\title{
MECHANISMS OF INFLUENCE OF UNBALANCED FEEDING ON MORPHO-FUNCTIONAL STATE OF LIVER IN PREGNANT RATS
}

\author{
Milena Kuznetsova \\ Assistant at the Department of General and Clinical Pathophysiology \\ named after D. E. Alpern, Kharkiv National Medical University, Ukraine \\ e-mail: mkusya1605@gmail.com,orcid.org/0000-0002-2629-0662
}

Iryna Kuznetsova

Assistant at the Department of Medical Biology, Kharkiv National Medical University, Ukraine e-mail: kusya388@gmail.com,orcid.org/0000-0001-9745-5762

\section{Summary}

Pathology of the organs of the hepatobiliary system is one of the leading causes of death in highly developed countries. Among various factors that can negatively affect the morphofunctional state of the liver in the mother-fetus system, the alimentary factor occupies the first place.

The aim of this study was to establish the mechanisms of influence of excess nutrients in the diet on the morphology and functional state of the liver of pregnant rats.

The structural and functional state of the liver of 7 rats that received an excess diet during pregnancy and 6 rats of the control group were studied.

Morphological changes were manifested by uneven expression of eNOS and a positive reaction of iNOS, which was observed not only in macrophages, but also in single endotheliocytes of dilated sinusoids. Functional changes were characterized by the development of dyslipidemia in the blood serum and the accumulation of TG and NEFA in the liver tissues.

The data obtained in the course of the study may indicate pronounced disorders of lipid metabolism, which can be regarded as a risk factor for the development of steatosis, liver fibrosis, atherosclerosis and type 2 diabetes mellitus.

Keywords: structural and functional state of liver, pregnant rats, excess diet.

DOI: https://doi.org/10.23856/4532

\section{Introduction}

In recent decades, there has been a tendency towards an increase in the proportion of diseases of the hepatobiliary system in the structure of somatic pathology among pregnant women in the highly developed countries of Europe. One of the key reasons that can have a negative impact on the structural and functional state of the liver of the mother and the fetus is the alimentary factor (diet with excess of nutrients).

In particular, it was proved that a high-fat diet can lead to the development of steatosis of varшув degree of severity, inflammation, and fibrosis, which in turn is determined by the nature of the diet (percentage and composition of lipids). According to (Jou J et al., 2008: 370-3), the $10 \%$ of vegetable oil, lard and $2 \%$ of cholesterol from the total composition of the diet in rats for 12 weeks leads to fatty infiltration of liver and to formation of steatosis in non-pregnant rats (Jou, J. et al., 2008: 371). The authors (Bivalkevich, N.V. et al., 2015: 39) found that prolonged exposure for 180 days to a high-lipid diet (19\% rendered beef tallow and $2 \%$ cholesterol) led to 
the development of steatohepatitis and triggered the fibrogenesis processes. It has been proven that a high-lipid diet leads to intensification of lipid peroxidation (LPO) processes and depletion of antioxidant defense mechanisms (Bivalkevich, N.V. et al., 2015: 41).

In addition, the effect from excess fat in diet in the occurrence of obesity and non-alcoholic fatty liver disease (NAFLD) has been proven. It was found that in young rats the induction of obesity by a diet with excess lipids is more effective than in rats of older age (Rossi, A. et al., 2020; Derks, N.G. et al., 2015: 537). The scientific works of (de Castro, U.G.M. et al., 2013) proved that young 4-week-old Fischer rats fed based on a diet enriched with animal fat (lard $40 \%$ by weight) had an increase in body weight and abdominal obesity (de Castro, U.G.M. et al., 2013). Literature data indicate that excess fat in the diet of rodents leads to the development of hypertrophy and fibrosis of the heart, myocardial necrosis, while in mice it also induces an increase in systolic blood pressure and the development of endothelial dysfunction (Francini-Pesenti, F. et al., 2019; Brambilla, A. et al., 2014: 92). (Senaphan, K. et al., 2013: 211) proved the short-term effect of a combined high-calorie diet (milk fat $21 \%$ and sucrose $34 \%$ by weight) on the induction of metabolic syndrome in 8-week-old $\mathrm{C} 57 \mathrm{~B} \alpha / 6$ mice and the development of the expression of genes involved in lipid metabolism and genes involved in transmission of insulin signal to tissue cells (Senaphan, K. et al., 2013:212).

Despite the presence of studies dedicated to the study of influence of the alimentary factor on the structure and functional state of the rat liver, the issues of its effect in the mother-fetus system remain underresearched.

The aim of this work was to study the mechanisms of influence of an excess diet on the structural and functional state of the liver in pregnant rats.

\section{Materials and methods}

The experimental work was carried out in 13 heads of 4-month-old female rats of the WAG population, which were divided into two groups: 7 female rats, which received the basic vivarium during pregnancy (group 1 or control group); 6 females, which received a diet with excess nutrients during pregnancy (group 2 or main group). The simulation of the effect of the alimentary factor on rats was carried out using an experimental model developed by the staff of the Department of General and Clinical Pathophysiology named after D.E. Alpern, at the Kharkiv National Medical University (Nikolaeva, O.V., Kovaltsova, M.V., Yevtushenko, T.G. (2013). Pat. 80979 Ukraine IPC G09B 23/28 (2006.01) Method of excess weight modeling).

\subsection{Immunohistochemical research}

The morphofunctional state of the organ was assessed by the results of immunohistochemical (IHC) studies of liver tissue and biochemical analysis of blood serum and organ homogenates. To assess the state of the liver vascular endothelium by the IHC method, a qualitative reaction was carried out to determine the expression of markers of nitric oxide metabolism: endothelial nitric oxide synthase (eNOS) and inducible nitric oxide synthase (iNOS). We used concentrated polyclonal rabbit antibodies (PCAT) from Thermo scientific (Germany) - Nitric Oxide Synthase inducible (iNOS) Rabbit Polyclonal Antibody dissolved at 1:100, and Nitric Oxide Synthase endothelial (eNOS) Rabbit Polyclonal Antibody dissolved at 1:50. 


\subsection{Biochemical research}

The functional state of the liver was assessed by the level of lipid metabolism indicators: total cholesterol (CS), low density lipoproteins (LDL), very low density lipoproteins (VLDL), high density lipoproteins (HDL), triglycerides (TG), atherogenic index (AI). The level of cholesterol, triglycerides, and HDL was determined by the spectrophotometric method using the reagent kits by Olvex (Russia), while the level of LDL, VLDL, and AI - by the calculation method. The fractional composition of lipids (cholesterol - CS, phospholipids - PL, triglycerides - TG, non-esterified fatty acids - NEFA) was studied in the liver tissue by thin layer chromatography on Silufol plates (Kamyshnikov, V.S., 2016).

All procedures on animals were performed in compliance with the rules and international recommendations of the European Convention for the Protection of Vertebrate Animals used for experiments or other scientific purposes (Strasbourg, 1986). Animals were withdrawn from the experiment by decapitation immediately after the birth of offspring. Statistical processing of the research results was carried out using the GraphPadPrism5 software. The Mann-Whitney $\mathrm{U}$ criterion was used to determine the significance of differences.

\section{Results and discussion}

The IHC study of the liver tissue of rats of the main group revealed changes, which consisted in the fact that the distribution of endothelial synthase in the organ of rats of the Group 2 was somewhat uneven, intermittent, with an overall moderate marker reaction.

A positive iNOS reaction in the observations of this group was detected not only in macrophages, but also in single endotheliocytes of dilated sinusoids, endothelial cells of the central vein, and in single isolated hepatocytes (Fig. 1).

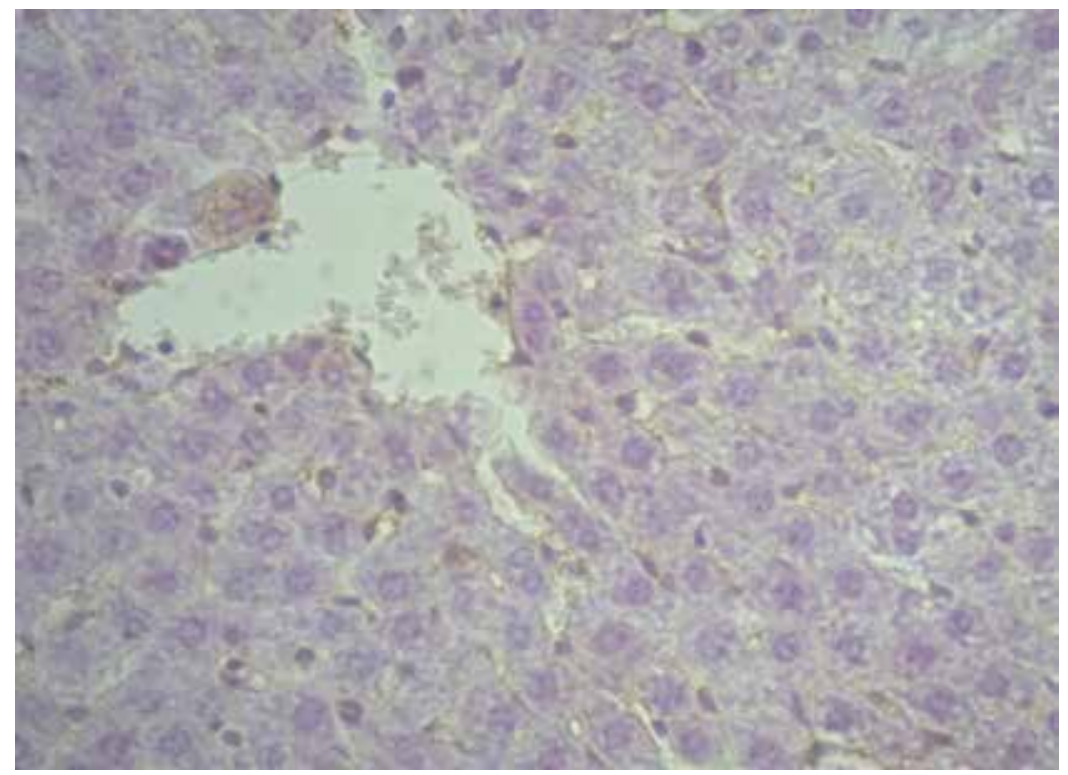

Fig. 1. Liver of rats, Group 2. Positive staining of endothelial cells and single hepatocytes. Reaction with PCAT to anti-iNOS, $\times 400$ 
The revealed changes indicated a minimal expression of eNOS, which indicated high compensatory capabilities of the mother's body during consumption of a diet with excess nutrients. The accumulation of iNOS promoted increased damage to hepatocytes, which in turn lead to the death of endothelial cells and the progression of sclerosis of the connective tissue structures of the liver. These immunomorphological features confirmed the presence of a moderately pronounced degree of endothelial dysfunction, which led to a decrease in the functional activity of the organ.

The Figures 2 and 3 present the results of biochemical studies of blood serum and liver homogenates needed to assess the functional state.

\section{Biochemical parameters of lipid exchange in blood serum of Group 2 mother rats}

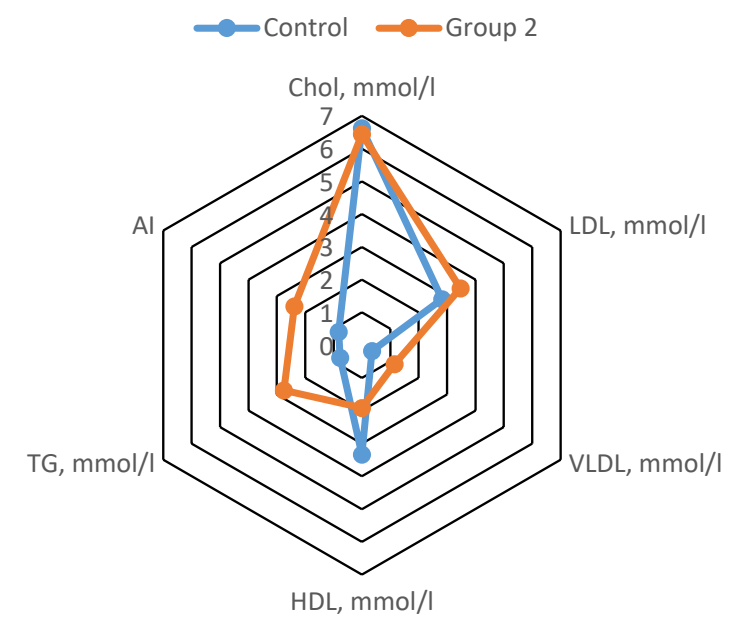

Fig. 2

As can be noted according to the data presented in Fig. 2, the lipid profile of mother rats of Group 2 contains the level of cholesterol corresponding to that in rats of the control group, and TG is significantly increased - by $257.1 \%, \mathrm{p}<0.05$. Moreover, a significant increase of AI - by $190.2 \%$ (p 0.001) was detected, which was due to an increase in VLDL by $219.4 \%$ and a decrease in HDL by $42.6 \%(\mathrm{p}<0.05)$. The established regularities of the lipid profile indicate pronounced disorders of lipid metabolism and prove a high degree of risk of atherosclerosis in females who received an alimentary excess of nutrients in the diet.

The study of liver homogenates (Fig. 3) in rats of Group 2 revealed significant changes in the fractional composition of lipids, which were manifested by an increase in TG and NEFA by $71.7 \%$ and $95.1 \%(\mathrm{p}<0.01)$, respectively, at a PL level which did not differ from the control, and, a decrease in the level of cholesterol - by $28.1 \%(\mathrm{p}<0.01)$.

The revealed changes indicate the presence of a pronounced degree of lipid metabolism disorders, which can serve as a risk factor for the development of metabolic syndrome, atherosclerosis and various organic liver pathologies.

The morphofunctional changes revealed in the rat liver can be explained by the fact that the consumption of a diet with excess nutrients leads to a functional overload of hepatocytes. 
Long-term functional overload of liver cells potentiates degenerative changes in liver cells, which subsequently leads to their depletion. Furthermore, these changes contribute to the activation of the stress response, and this, in turn, contributes to hypercatecholaminemia, which aggravates liver damage.

\section{Fractional composition of lipids in liver homogenates of Group 2 mother rats}

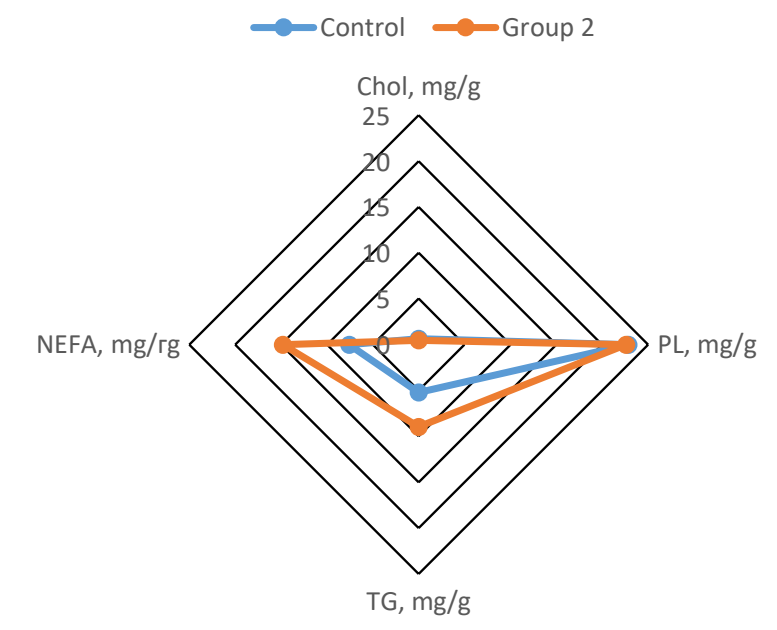

Fig. 3

\section{Conclusions}

1. Long-term consumption of excess nutrients in the diet leads to the development of structural and functional disorders, which were manifested by a positive reaction of inducible NO-synthase, which can become a risk factor for the development of fibrotic changes in the liver.

2. Functional changes in the liver were manifested by the development of dyslipidemia (significant increase in TG, VLDL and AI), which can be regarded as a risk factor for the development of atherosclerosis, metabolic syndrome and type 2 diabetes mellitus.

3. Biochemical studies of liver homogenates indicated violations of lipid metabolism, which was confirmed by the accumulation of TG and NEFA with a decrease in the level of CS and PL in the tissue of the organ, which can be regarded as a predisposing risk factor for the development of steatohepatitis, hepatic steatosis and other organic liver pathologies.

\section{References}

Jou, J., Choi, S., Diehl, A. (2008). Mechanisms of disease progression in nonalcoholic fatty liver disease. Semin Liver Dis, 28, 370-379.

Bivalkevich, N.V., Denisenko, Yu.K., Novgorodtseva T.P. (2015). Methodological approaches to experimental modeling of non-alcoholic fatty liver disease. RZHGGK, 4, 39-43. 
de Castro, U.G.M., dos Santos, R.A.S., Silva, M.E. et al. (2013). Age dependent effect of high-fructose and high-fat diets on lipid metabolism and lipid accumulation in liver and kidney of rats. Lipids in health and disease, 12(136), 1476-511X.

Nikolaeva, O.V., Kovaltsova, M.V., Yevtushenko, T.G. (2013). Pat. 80979 Ukraine IPC G09B 23/28 (2006.01) Method of excess weight modeling / applicant and patent holder - Kharkiv National Medical University. - No. u 2013 01221; declared 01.02.2013; publ. 10.06.2013, Bul. No. 11.

Kamyshnikov, V.S. (2016). Methods of clinical laboratory research. Moscow: Medipress Inform publishers, $736 \mathrm{p}$.

Senaphan, K., Boonla, O., Timinkul, A. et al. (2013). Effect of feluric acid on high-carbohydrate, high-fat diet induced metabolic syndrome in rats. Srinagarind Med J, 28, 211-214.

Rossi, A., Hoogeveen, I.J., Bastek, V.B. et al. (2020). Dietary lipids in glycogen storage disease type III: A systematic literature study case studies and future recommendations. J Inherit Metab Dis, 43(4), 770-777. DOI:http://doi.org/10.1002/jimd.12224.

Francini-Pesenti, F., Tresso, S., Vitturi, N. (2019). Modified Atkins ketogenic diet improves heart and skeletal muscle function in glycogen storage disease type III. Acta Myol, 38(7), 17-20. Derks, T.G., van Rijn, M. (2015). Lipids in hepatic glycogen storage diseases: pathophysiology, monitoring of dietary management and future directions. J Inherit Metab Dis, 38(3), 537-543. DOI:http://doi.org/10.1007/s10545-015-9811-2.

Brambilla, A., Mannarino, S., Pretese, R., Gasperini, S., Galimberti, C., Parini, R. (2014). Improvement of Cardiomyopathy after high-fat diet in two siblings with glycogen storage disease type III. JIMD Rep, 17, 91-95. 